\title{
Association of Systolic Blood Pressure with Progression of Symptomatic Intracranial Atherosclerotic Stenosis
}

\author{
Jong-Ho Park, ${ }^{a}$ Bruce Ovbiagele, ${ }^{\mathrm{b}}$ Keun-Sik Hong, ${ }^{\mathrm{c}}$ Sun U. Kwon ${ }^{\mathrm{d}}$

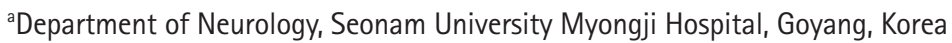 \\ bepartment of Neurology, Medical University of South Carolina, Charleston, SC, USA \\ 'Department of Neurology, Ilsan Paik Hospital, Inje University, Goyang, Korea \\ ${ }^{\mathrm{d} D e p a r t m e n t}$ of Neurology, Asan Medical Center, University of Ulsan College of Medicine, Seoul, Korea
}

Background and Purpose Elevated blood pressure (BP) is associated with greater severity of intracranial atherosclerotic stenosis (ICAS) and increased risk of ischemic stroke. Because little is known about the relationship of maintained BP level with progression of symptomatic ICAS (sICAS), we evaluated the independent association of maintained systolic BP (SBP) with risk of sICAS progression.

Methods We analyzed the Trial of cilOstazol in Symptomatic intracranial Stenosis 2, which evaluated 402

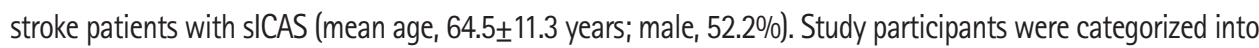
four groups according to mean SBP level: low-normal (<120 mm Hg), normal to high-normal (120 to $139 \mathrm{~mm}$ $\mathrm{Hg}$ ), high (140 to $159 \mathrm{~mm} \mathrm{Hg}$ ), and very-high ( $\geq 160 \mathrm{~mm} \mathrm{Hg}$ ). Progression of sICAS was defined as worsening in the degree of stenosis by $\geq 1$ grade on the 7-month magnetic resonance angiography follow-up.

Results sICAS progression was observed in $52(12.9 \%)$ subjects. Percentages of sICAS progression by mean SBP category showed a J-shape pattern: low-normal (21.4\%), normal to high-normal (10.7\%), high (11.4\%), and very-high (38.9\%). In multivariable analysis, compared to the normal to high-normal SBP group, odds ratios (95\% confidence intervals) were low-normal, 1.88 (0.62-5.67); high, 1.06 (0.47-2.37); and very-high, 8.75 (2.57-29.86). Rate of sICAS progression by $10-\mathrm{mm} \mathrm{Hg}$ strata showed a similar pattern to findings from mean SBP category $(9.47 ; 2.58-34.73$ for SBP $\geq 160 \mathrm{~mm} \mathrm{Hg})$.

Conclusions Among individuals with a recent ICAS stroke, very-high SBP level during the short-term period after the index stroke was associated with significantly greater odds of sICAS progression.
Correspondence: Keun-Sik Hong Department of Neurology, Ilsan Paik Hospital, Inje University, 170 Juhwa-ro, Ilsanseo-gu, Goyang 10380, Korea

Tel: $+82-31-910-7680$

Fax: +82-31-910-7368

E-mail: nrhks@paik.ac.kr

Co-correspondence: Sun U. Kwon Department of Neurology, Asan Medical Center, University of Ulsan College of Medicine, 88 Olympic-ro 43-gil, Songpa-gu, Seoul 05505, Korea

Tel: +82-2-3010-3960

Fax: +82-2-474-4691

E-mail: sukwon@amc.seoul.kr

Received: January 18, 2017

Revised: August 18, 2017

Accepted: August 21, 2017

The authors have no financial conflicts of interest.

Keywords Intracranial; Stenosis; Progression; Symptomatic; Blood pressure

\section{Introduction}

Intracranial atherosclerotic stenosis (ICAS) is one of the most common causes of stroke globally, as well as in Asia. ${ }^{1,2}$ Symptomatic ICAS (sICAS) is associated with a higher risk of recurrent stroke compared with other stroke subtypes. ${ }^{3}$ Progression of sICAS is associated with an increased risk of recurrent stroke ${ }^{4,5}$ and the risk of recurrent stroke in the sICAS territory increases monotonically with a higher degree of stenosis. ${ }^{6}$

On the basis of reduced capacity of cerebral autoregulation in patients with high blood pressure (BP), physicians have maintained high BP in patients after a sICAS stroke due to concern of the risk of hypoperfusion ischemia with BP-lowering therapy. However, allowing higher BP of $\geq 140 / 90 \mathrm{~mm} \mathrm{Hg}$ was not protective against stroke and actually increased the risk of stroke in the sICAS territory, and stroke overall.? For extracranial carotid stenosis, high BP was associated with increased risk of stroke in patients who had bilateral stenosis $<70 \%$ or unilateral stenosis $>70 \%$, although the relationship was reversed in patients with bilateral stenosis $>70 \% .^{8}$ In an- 
other study, BP lowering therapy reduced the progression of extracranial carotid stenosis in patients with hypertension. ${ }^{9}$ With regard to ICAS, an earlier autopsy study showed that high diastolic BP was associated with atherosclerosis in the large intracranial arteries. ${ }^{10}$ Furthermore, a post hoc analysis of the Warfarin-Aspirin Symptomatic Intracranial Disease (WASID) trial, high SBP ( $\geq 160 \mathrm{~mm} \mathrm{Hg}$ ) was associated with increased risk of stroke in the sICAS territory. However, the association between BP control level and sICAS progression has not been well studied.

This exploratory study, analyzing clinical trial data of patients with sICAS, evaluated the independent association of systolic BP (SBP) maintained at low level vs. high level with the risk of sICAS progression among subjects after an ICAS stroke.

\section{Methods}

\section{Study subjects and database}

This is a post hoc observational analysis of data from the Trial of cilOstazol in Symptomatic intracranial atherosclerotic Stenosis 2 (TOSS-2), which enrolled 457 patients with ischemic stroke due to sICAS, involving the M1 segment of the middle cerebral artery (MCA) or the basilar artery (BA), within 2 weeks of symptom onset and followed for 7 months. ${ }^{11}$ The TOSS-2 trial was a randomized, double-blind, multicenter trial performed at 20 centers in four East Asian countries. The main objective of the study was to compare the efficacy of cilostazol plus aspirin vs. clopidogrel plus aspirin on the progression of sICAS assessed by magnetic resonance angiography (MRA) obtained at the 7-month follow-up visit."1 Demographic, clinical, and laboratory data, including BP recordings, were collected at randomization, with subsequent clinical and laboratory data obtained during follow-up visits at 1, 3, 5, and 7 months. During the trial, physicians were recommended to provide aggressive risk factor control, including statin therapy. Serum lipid profile, glycosylated hemoglobin (HbA1c) level, and MRA were obtained at baseline and at the 7-month follow-up. Subjects who had no follow-up MRA or post baseline SBP data were excluded. All participants provided written informed consent, and the trial was approved by the ethics committees of all participating centers.

\section{Definition of stenosis and outcome measures}

The primary endpoint was the progression of sICAS on the follow-up MRA. Follow-up MRA was obtained at the 7-month follow-up visit or at the time of patient withdrawal. The severity of stenosis in the MCAs and BA of each subject was classified by the imaging review committee, blinded to clinical infor- mation and the location of the symptomatic stenosis, into 1 of 5 grades: normal, mild (signal reduction $<50 \%$ ), moderate (signal reduction $\geq 50 \%$ ), severe (focal signal loss with the presence of distal flow), and occlusion (sudden cutoff without distal flow void). The progression of sICAS was defined as worsening in the degree of stenosis by $\geq 1$ grade on the follow-up MRA compared to the baseline MRA, while the regression of sICAS was defined as improvement in the degree of stenosis." The secondary endpoint was the recurrence of any ischemic stroke.

\section{Statistics}

On the basis of the Seventh Report of the Joint National Committee on Prevention, Detection, Evaluation, and Treatment of High Blood Pressure (JNC 7), the classification of normal BP, prehypertension, stage 1 hypertension, and stage 2 hyperten$\operatorname{sion}^{12}$ study participants were categorized into four groups according to mean SBP level during the follow-up: low-normal ( $<120 \mathrm{~mm} \mathrm{Hg}$ ), normal to high-normal (120 to $139 \mathrm{~mm} \mathrm{Hg}$ ), high (140 to $159 \mathrm{~mm} \mathrm{Hg})$, and very-high ( $\geq 160 \mathrm{~mm} \mathrm{Hg}$ ). The group with SBP of 120 to $139 \mathrm{~mm} \mathrm{Hg}$ served as the reference group, since the target SBP level for ICAS stroke is less than $140 \mathrm{~mm} \mathrm{Hg} .{ }^{3}$ The mean SBP category was chosen for consistency with other studies. ${ }^{7,13}$ Additionally, low-normal SBP range was associated with increased risk of recurrent stroke in patients with ischemic stroke of various subtypes. ${ }^{13}$ We also further explored the rate of sICAS progression by $10-\mathrm{mm} \mathrm{Hg}$ strata of mean SBP. Estimated odds ratios (ORs) were calculated with reference to SBP 130 to $139 \mathrm{~mm}$ Hg. Mean follow-up SBP from the baseline to final visit was calculated for each subject. If the patient did not fully visit during follow-up $(n=21)$ or experienced vascular event/death $(n=9)$ before the 7-month follow-up, the SBP measurement was censored at that point. Comparisons across the groups were examined using the $\chi^{2}$ test or Fisher's exact test for categorical variables and one-way analysis of variance, followed by the Dunnett's test for multiple comparisons, or paired $t$-test for comparison between baseline and follow-up SBP for continuous variables. Backward elimination multivariable logistic regression analyses were performed to estimate the association of SBP with the SICAS progression within the short term follow-up period as follows: (1) after adjusting for baseline covariates (age, male sex, serum levels of creatinine and homocysteine, hypertension, treatment for high $\mathrm{BP}$, renin-angiotensin system inhibitor use, and smoking [model I, $P<0.10$ in univariate analyses in Table 1]) and; (2) after adjusting for model I plus diabetes mellitus, HbA1c level, low-density lipoprotein cholesterol level, qualifying stroke severity, cilostazol plus aspirin medication (vs. clopidogrel plus 
aspirin), and statin medication that are additionally related to cardiovascular recurrence or death (model II). Model fit was tested using modifications of methods by Hosmer and Lemeshow. ${ }^{14}$ Cox proportional hazard regression analyses were performed to estimate the risk of ischemic stroke. Results are expressed as OR for sICAS progression or as hazard ratio (HR) for ischemic stroke with 95\% confidence intervals (Cls). The interaction between cilostazol (vs. clopidogrel) and each SBP level on sICAS progression was assessed by including the appropriate interaction terms in the model. All analyses were conducted using IBM SPSS version 22.0 (IBM Corp., Armonk, NY, USA). A probability value of $<0.05$ was considered statistically significant.

\section{Results}

\section{Subjects characteristics by mean SBP category}

A total of 402 participants (mean age, $64.5 \pm 11.3$ years; male, $52.2 \%$ ) were included in this study after excluding 55 subjects; 48 subjects did not have a follow-up magnetic resonance imaging and 7 subjects had no follow-up BP data. Mean baseline SBP of study subjects was $140.8 \pm 22.8 \mathrm{~mm} \mathrm{Hg}$. The frequency of subjects by $10-\mathrm{mm} \mathrm{Hg}$ strata of mean SBP is shown in Figure 1. Table 1 presents baseline demographic and clinical characteristics across the mean SBP level categories. Specifically, when compared with the normal to high-normal group, the very-high SBP group had higher frequencies of hypertension, antihypertensive medication and renin-angiotensin inhibitor use (angiotensin receptor blocker or angiotensin-converting enzyme inhibitor), and higher serum levels of creatinine and

Table 1. Baseline demographic and clinical characteristics of 402 subjects across SBP category

\begin{tabular}{|c|c|c|c|c|c|}
\hline & \multicolumn{4}{|c|}{ Mean SBP category $(\mathrm{mm} \mathrm{Hg})$} & \multirow[b]{2}{*}{$P$} \\
\hline & $\begin{array}{l}\text { Low-normal } \\
\qquad<120)\end{array}$ & $\begin{array}{l}\text { Normal to high-normal } \\
(120 \text { to } 139)\end{array}$ & $\begin{array}{l}\text { High } \\
\text { (140 to 159) }\end{array}$ & $\begin{array}{l}\text { Very-high } \\
(\geq 160)\end{array}$ & \\
\hline Number of subjects & 28 & 233 & 123 & 18 & \\
\hline Age (years) & $62.2 \pm 9.7$ & $64.2 \pm 11.4$ & $65.4 \pm 11.6$ & $66.9 \pm 11.2$ & 0.413 \\
\hline Glucose (mg/dL) & $123.6 \pm 46.7$ & $139.1 \pm 68.9$ & $149.3 \pm 69.2$ & $132.5 \pm 38.7$ & 0.242 \\
\hline HbA1c (\%) & $6.9 \pm 2.3$ & $6.6 \pm 1.7$ & $7.0 \pm 2.0$ & $6.5 \pm 1.2$ & 0.364 \\
\hline Total cholesterol (mg/dL) & $180.0 \pm 36.6$ & $190.5 \pm 41.2$ & $198.4 \pm 44.2$ & $184.3 \pm 55.9$ & 0.130 \\
\hline LDL-C (mg/dL) & $119.9 \pm 27.0$ & $122.1 \pm 35.8$ & $121.0 \pm 35.8$ & $115.9 \pm 55.0$ & 0.904 \\
\hline $\mathrm{HDL}-\mathrm{C}(\mathrm{mg} / \mathrm{dL})$ & $40.0 \pm 12.0$ & $43.8 \pm 11.6$ & $44.9 \pm 12.6$ & $44.7 \pm 13.2$ & 0.275 \\
\hline Creatinine (mg/dL) & $1.01 \pm 0.26$ & $0.89 \pm 0.26$ & $0.92 \pm 0.33$ & $1.06 \pm 0.34$ & 0.032 \\
\hline Homocysteine ( $\mu \mathrm{mol} / \mathrm{L})$ & $16.4 \pm 11.1^{*}$ & $11.9 \pm 6.0^{*}$ & $12.8 \pm 9.9$ & $17.4 \pm 15.5$ & 0.009 \\
\hline Qualifying stroke NIHSS & $2.5 \pm 3.5$ & $3.0 \pm 3.0$ & $3.6 \pm 2.6$ & $3.7 \pm 3.1$ & 0.154 \\
\hline Time from qualifying & $8.2 \pm 2.7$ & $8.0 \pm 2.5$ & $8.0 \pm 2.5$ & $8.1 \pm 2.8$ & 0.987 \\
\hline \multicolumn{6}{|l|}{ event to randomization (day) } \\
\hline Male & $23(82.1)$ & $127(54.5)$ & $50(40.7)$ & $10(55.6)$ & 0.001 \\
\hline Hypertension & $12(42.9)$ & $151(64.8)$ & $107(87.0)$ & $17(94.4)$ & $<0.001$ \\
\hline Treatment for high BP & $17(60.7)$ & $162(69.5)$ & 120 (97.6) & $17(94.4)$ & $<0.001$ \\
\hline RAS inhibitor ${ }^{\dagger}$ & $13(46.4)$ & $92(39.5)$ & $85(69.1)$ & $16(88.9)$ & $<0.001$ \\
\hline Calcium channel blocker & $3(10.7)$ & $90(38.6)$ & $83(67.5)$ & $12(66.7)$ & $<0.001$ \\
\hline Others & $9(32.1)$ & $96(41.2)$ & $71(57.7)$ & $10(55.6)$ & 0.008 \\
\hline Diabetes mellitus & $12(42.9)$ & $90(38.6)$ & $56(45.5)$ & $9(50.0)$ & 0.538 \\
\hline Current smoker & $20(71.4)$ & $110(47.2)$ & $44(35.8)$ & $3(16.7)$ & $<0.001$ \\
\hline Family history of stroke & $6(21.4)$ & $61(26.2)$ & 37 (30.1) & $2(11.1)$ & 0.336 \\
\hline Cilostazol group ${ }^{\ddagger}$ & $12(42.9)$ & $115(49.4)$ & $70(56.9)$ & $6(33.3)$ & 0.178 \\
\hline On statin treatment & $21(75.0)$ & $159(68.2)$ & 89 (72.4) & $12(66.7)$ & 0.779 \\
\hline
\end{tabular}

Values are presented as number (\%) or mean \pm standard deviation, as appropriate, otherwise stated.

SBP, systolic blood pressure; HbA1c, glycosylated hemoglobin; LDL-C, low-density lipoprotein cholesterol; HDL-C, high-density lipoprotein cholesterol; NIHSS, National Institutes of Health Stroke Scale; RAS, renin-angiotensin system.

*Indicates significant difference between them $(P=0.021)$ by Dunnet post hoc tests; ${ }^{+}$Includes angiotensin receptor blocker or angiotensin-converting enzyme inhibitor; ${ }^{\ddagger}$ Aspirin plus cilostazol. 


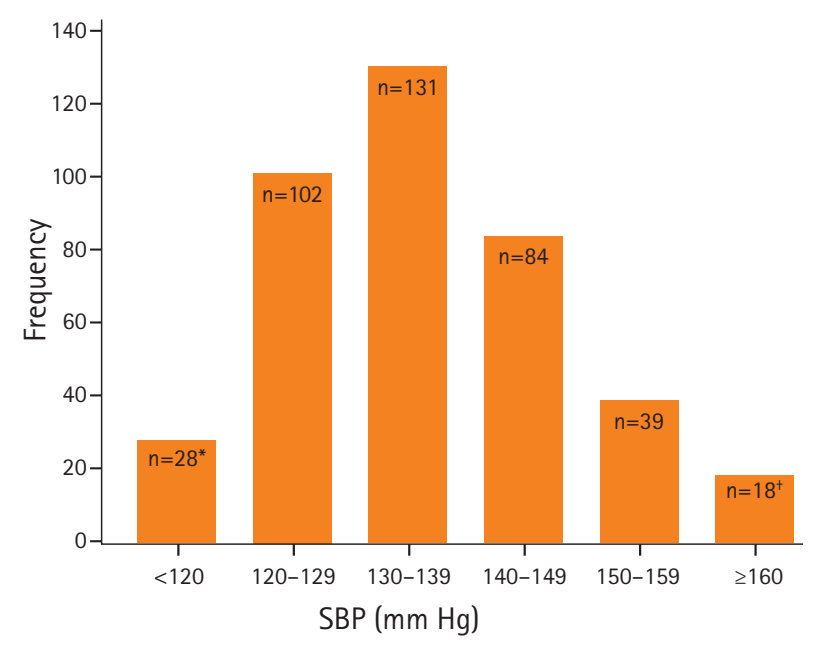

Figure 1. The frequency of subjects by $10-\mathrm{mm} \mathrm{Hg}$ strata of mean systolic blood pressure (SBP). *Includes SBP $<110 \mathrm{~mm} \mathrm{Hg}(\mathrm{n}=3)$, SBP 110-119 mm $\mathrm{Hg}(n=25) .{ }^{+}$Includes SBP 160-169 mm Hg $(n=10)$, SBP $170-179 \mathrm{~mm} \mathrm{Hg}$ $(n=4)$, SBP $180-189 \mathrm{~mm} \mathrm{Hg}(n=1)$, SBP $190-199 \mathrm{~mm} \mathrm{Hg}(n=2)$, and SBP 200-209 mm Hg $(n=1)$. homocysteine, whereas the frequency of current smoking was lower.

\section{Baseline sICAS by mean SBP category}

Neither the severity nor distribution of baseline sICAS differed across the mean SBP categories (Supplementary Tables 1 and 2). After 7-month treatment, a total of 52 (12.9\%) sICAS progressions and $12(3.0 \%)$ ischemic strokes were recorded. Table 2 shows baseline, follow-up and change in the BP levels, and serum variables, including cholesterol and $\mathrm{HbA} 1 \mathrm{c}$ levels, by mean SBP category. Mean baseline and follow-up BPs were lowest in the low-normal SBP level group, but the mean BP change was not significantly different across the mean SBP categories. Supplementary Table 3 displays comparisons of SBP between baseline and follow-up by each mean SBP category, and shows that mean SBP levels were significantly lower at follow-up in the high SBP group ( $P=0.002$ by paired $t$-test). There were no differences for the other SBP groups. Outcomes

Table 2. Baseline, follow-up, and change in BP levels and serum variables by mean SBP category in 402 patients with a recent ICAS stroke

\begin{tabular}{|c|c|c|c|c|c|}
\hline & \multicolumn{4}{|c|}{ Mean SBP category $(\mathrm{mm} \mathrm{Hg})$} & \multirow{2}{*}{$P$} \\
\hline & $<120(n=28)$ & 120 to $139(n=233)$ & 140 to $159(n=123)$ & $\geq 160(n=18)$ & \\
\hline \multicolumn{6}{|l|}{$\mathrm{BP}(\mathrm{mm} \mathrm{Hg})$} \\
\hline \multicolumn{6}{|l|}{ At baseline } \\
\hline Systolic & $121.5 \pm 14.0^{*}$ & $133.1 \pm 15.8^{*}$ & $154.7 \pm 22.8$ & $175.4 \pm 27.0$ & $<0.001$ \\
\hline Diastolic & $75.8 \pm 7.7^{*}$ & $79.9 \pm 10.6$ & $85.1 \pm 11.4^{*}$ & $92.6 \pm 13.8$ & $<0.001$ \\
\hline \multicolumn{6}{|l|}{ At follow-up } \\
\hline Systolic & $112.8 \pm 11.0^{*}$ & $126.6 \pm 12.6^{*}$ & $143.9 \pm 15.6$ & $167.8 \pm 26.1$ & $<0.001$ \\
\hline Diastolic & $67.6 \pm 7.1^{*}$ & $75.6 \pm 9.7^{*}$ & $81.3 \pm 9.8$ & $93.9 \pm 13.9$ & $<0.001$ \\
\hline \multicolumn{6}{|l|}{ Change in level } \\
\hline Systolic & $-8.7 \pm 16.5$ & $-6.4 \pm 21.0$ & $-10.8 \pm 31.0$ & $-7.7 \pm 38.7$ & 0.474 \\
\hline Diastolic & $-8.1 \pm 9.5$ & $-4.3 \pm 13.2$ & $-3.8 \pm 15.5$ & $1.3 \pm 20.9$ & 0.174 \\
\hline \multicolumn{6}{|l|}{ LDL-C (mg/dL) } \\
\hline At baseline & $119.9 \pm 27.0$ & $122.1 \pm 35.8$ & $121.0 \pm 35.8$ & $115.9 \pm 55.0$ & 0.904 \\
\hline At follow-up & $102.7 \pm 36.2$ & $94.2 \pm 29.4$ & $94.2 \pm 34.3$ & $101.9 \pm 44.8$ & 0.473 \\
\hline Change in level & $-17.3 \pm 43.3$ & $-27.9 \pm 40.8$ & $-26.1 \pm 47.8$ & $-11.0 \pm 33.6$ & 0.336 \\
\hline \multicolumn{6}{|l|}{$\mathrm{HDL}-\mathrm{C}(\mathrm{mg} / \mathrm{dL})$} \\
\hline At baseline & $40.0 \pm 12.0$ & $43.8 \pm 11.6$ & $44.9 \pm 12.6$ & $44.7 \pm 13.2$ & 0.275 \\
\hline At follow-up & $44.8 \pm 10.9$ & $48.7 \pm 12.2$ & $50.4 \pm 14.0$ & $51.9 \pm 16.6$ & 0.157 \\
\hline Change in level & $4.8 \pm 12.3$ & $4.9 \pm 10.9$ & $5.3 \pm 12.5$ & $7.4 \pm 12.3$ & 0.871 \\
\hline \multicolumn{6}{|l|}{ HbA1c (\%) } \\
\hline At baseline & $6.9 \pm 2.3$ & $6.6 \pm 1.7$ & $7.0 \pm 2.0$ & $6.5 \pm 1.2$ & 0.364 \\
\hline At follow-up & $6.7 \pm 1.6$ & $6.2 \pm 1.1$ & $6.3 \pm 1.1$ & $6.1 \pm 0.9$ & 0.120 \\
\hline Change in level & $-0.2 \pm 1.9$ & $-0.4 \pm 1.3$ & $-0.6 \pm 1.8$ & $-0.3 \pm 0.9$ & 0.529 \\
\hline
\end{tabular}

Values are presented as number (\%) or mean \pm standard deviation.

SBP, systolic blood pressure; ICAS, intracranial atherosclerotic stenosis; LDL-C, low-density lipoprotein cholesterol; HDL-C, high-density lipoprotein cholesterol; $\mathrm{HbA1C}$, glycosylated hemoglobin.

${ }^{*}$ Indicates significant difference between them $(P<0.01)$ by Dunnet post hoc tests. 
of sICAS at 7-month follow-up differed across the mean SBP categories (Table 3). When compared with normal to high-normal group, the very-high SBP group was more likely to have slCAS progression and less likely to have sICAS regression $(P=0.009)$.

\section{Associations of mean SBP level with sICAS progression}

Table 4 provides unadjusted and adjusted analyses for sICAS progression and ischemic stroke. In the unadjusted analysis, when referenced to the normal to high-normal SBP, very-high SBP was significantly associated with an increased risk of slCAS progression (OR 5.30; 1.88-14.90; $P=0.002$ ). In multivariable backward elimination regression analysis, compared with the normal to high-normal SBP, very-high SBP was associated with increased risk of sICAS progression (OR 8.36; 2.49-28.12,
$P=0.001$ in model I); the association remained constant after further adjustment (OR 8.75; 2.57-29.86, $P=0.001$ in model II). However, low-normal SBP showed a trend toward higher risk of sICAS progression (OR 1.87; $0.63-5.51$ in model I and 1.88; 0.62-5.67 in model II). Figure 2 depicts percentages of sICAS progression by mean SBP category, revealing a near-similar J-curve pattern to this relationship among the patients with a sICAS stroke. For the secondary outcome, only very-high SBP level was associated with increased risk of ischemic stroke in the univariate analysis (HR 6.99; $1.30-37.49 ; P=0.023)$, but this association lost significance after adjusting for covariates. The small sample size in the very-high SBP group might account for the statistical non-significance. Supplementary Table 4 provides the associations of SBP with the outcome events by $10-\mathrm{mm} \mathrm{Hg}$ strata of mean SBP. Compared with the SBP levels of 130 to $139 \mathrm{~mm} \mathrm{Hg}$, SBP levels of $\geq 160 \mathrm{~mm} \mathrm{Hg}$ was associ-

Table 3. Outcomes of baseline sICAS across mean SBP category

\begin{tabular}{|c|c|c|c|c|c|}
\hline \multirow{2}{*}{ sICAS outcome } & \multicolumn{4}{|c|}{ Mean SBP category $(\mathrm{mm} \mathrm{Hg})$} & \multirow{2}{*}{$P^{*}$} \\
\hline & $<120(n=28)$ & 120 to $139(n=233)$ & 140 to $159(n=123)$ & $\geq 160(n=18)$ & \\
\hline Regression & $9(32.1)$ & $62(26.6)$ & $37(30.1)$ & $1(5.6)$ & 0.009 \\
\hline Quiescence & $13(46.4)$ & $146(62.7)$ & 72 (58.5) & $10(55.6)$ & \\
\hline Progression & $6(21.4)$ & $25(10.7)$ & $14(11.4)$ & $7(38.9)$ & \\
\hline
\end{tabular}

Values are presented as number (\%) of patients.

sICAS, symptomatic intracranial atherosclerotic stenosis; SBP, systolic blood pressure.

*By $\chi^{2}$ test.

Table 4. ORs for the progression of sICAS and recurrent ischemic stroke by mean SBP category

\begin{tabular}{|c|c|c|c|c|}
\hline & & Mean SBP category & $\mathrm{m} \mathrm{Hg})$ & \\
\hline & Low-normal $(<120)$ & Normal to high-normal (120 to 139 ) & High (140 to 159$)$ & Very-high ( $\geq 160$ ) \\
\hline Number of subjects & 28 & 233 & 123 & 18 \\
\hline Primary outcome & OR $(95 \% \mathrm{Cl})$ & & OR (95\% Cl) & OR $(95 \% \mathrm{Cl})$ \\
\hline sICAS progression & $6(21.4)$ & $25(10.7)$ & $14(11.4)$ & $7(38.9)$ \\
\hline Unadjusted & $2.27(0.84-6.13)$ & 1 [Reference] & $1.07(0.53-2.14)$ & $5.30(1.88-14.90)$ \\
\hline Adjusted & & & & \\
\hline Model I ${ }^{\ddagger}$ & $1.87(0.63-5.51)$ & 1 [Reference] & $1.04(0.48-2.23)$ & $8.36(2.49-28.12)^{*}$ \\
\hline Model $\|^{\S}$ & $1.88(0.62-5.67)$ & 1 [Reference] & $1.06(0.47-2.37)$ & $8.75(2.57-29.86)^{*}$ \\
\hline Secondary outcome & $\mathrm{HR}(95 \% \mathrm{Cl})$ & & HR (95\% Cl) & HR $(95 \% \mathrm{Cl})$ \\
\hline Ischemic stroke" & $0(0)$ & $6(2.6)$ & $4(3.3)$ & $2(11.1)$ \\
\hline Unadjusted & $0.00(0.00-0.00)$ & 1 [Reference] & $1.51(0.41-5.63)$ & $6.99(1.30-37.49)^{+}$ \\
\hline Adjusted & & & & \\
\hline Model I ${ }^{\ddagger}$ & $0.00(0.00-0.00)$ & 1 [Reference] & $1.01(0.17-5.97)$ & $0.74(0.02-22.88)$ \\
\hline Model II ${ }^{\S}$ & $0.00(0.00-0.00)$ & 1 [Reference] & $0.77(0.11-5.35)$ & $0.66(0.02-20.05)$ \\
\hline
\end{tabular}

Values are presented as number (\%) unless otherwise indicated.

$\mathrm{OR}$, odds ratio; sICAS, symptomatic intracranial atherosclerotic stenosis; $\mathrm{SBP}$, systolic blood pressure; $\mathrm{Cl}$, confidence interval; $\mathrm{HR}$, hazard ratio.

${ }^{*} P=0.001 ;{ }^{\dagger} P=0.023 ;{ }^{\dagger}$ Adjusted for age, male sex, serum levels of creatinine and homocysteine, hypertension, treatment for high $\mathrm{BP}$, renin-angiotensin system modifier use, smoking as model I; ${ }^{\circledR}$ Adjusted for model I plus diabetes mellitus, glycosylated hemoglobin, low-density lipoprotein cholesterol, qualifying stroke severity, cilostazol+aspirin medication (vs. clopidogrel+aspirin), and statin medication; "Defined as any ischemic stroke or stroke in the sICAS territory. 


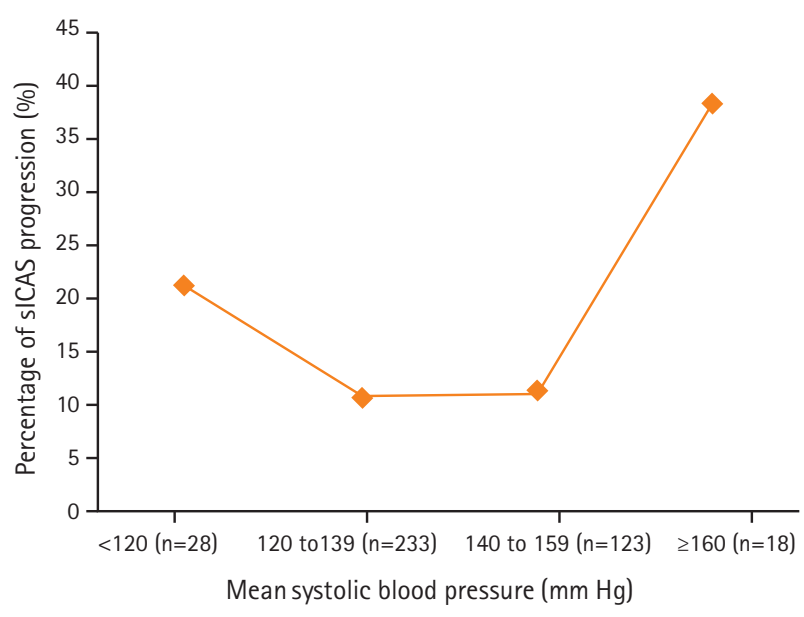

Figure 2. Percentage of sICAS progression by mean systolic blood pressure category among patients with an ICAS stroke. sICAS, symptomatic intracranial atherosclerotic stenosis.

ated with increased risk of sICAS progression (OR 8.53; 2.3830.62, $P=0.001$ in model I) and the association remained significant after further adjustment $(9.47 ; 2.58-34.73, P=0.001$ in model II). Low-normal SBP showed a trend toward greater odds of sICAS progression (OR 1.91; 0.60-6.09 in model I and $2.04 ; 0.62-6.71$ in model II). For ischemic stroke, compared with the SBP levels of 130 to $139 \mathrm{~mm} \mathrm{Hg}$, SBP levels of $\geq 160$ $\mathrm{mm} \mathrm{Hg}$ was linked to an increased risk of ischemic stroke in the univariate analysis (HR 7.33, 1.15-46.53; $P=0.035$ ), but this significance was not observed after adjusting for covariates. Another independent predictor of sICAS progression in the multivariable analyses (model II) was age (Supplementary Table 5). The interaction effect between cilostazol and each mean SBP level on sICAS progression was not significant (cilostazolxlow-normal SBP, $P=0.863$; cilostazolxhigh $S B P$, $P=0.051$; cilostazolxvery-high SBP, $P=0.889$; data not shown).

\section{Discussion}

In this analysis of 402 patients with a recent ischemic stroke related to sICAS, we observed that compared with a mean SBP of 120 to $139 \mathrm{~mm} \mathrm{Hg}$, very-high SBP (>160 mm Hg) was associated with more than an 8-fold increased risk of sICAS progression during a short-term period. These findings were independent of various risk factor treatments with antihypertensive drugs, antiplatelet agent (i.e., cilostazol) and statins. Furthermore, given that the severity and distribution of baseline sICAS were comparable across the mean SBP categories, very-high SBP during the follow-up may have contributed to the sICAS progression.

It is unclear how systolic BP exerts the development of sICAS progression by pathophysiological mechanism, but stenosis progression appears to be accompanied by a progression of atherosclerotic plaque. ${ }^{9}$ Conversely, the mitigation of sICAS progression might result from SBP lowering-induced changes in arterial wave propagation ${ }^{9}$ in addition to remodeling of atherosclerotic plaques by statins and by HbA1c control. ${ }^{15}$

In a post hoc of WASID trial, higher SBP ( $\geq 160 \mathrm{~mm} \mathrm{Hg}$, particularly) was associated with increased risk of stroke in the territory of the sICAS (HR 3.9, 95\% Cl 1.1-14.1), ${ }^{7}$ the finding of which conforms with our study showing that SBP level of $\geq 160$ $\mathrm{mm} \mathrm{Hg}$ was significantly linked to an increased risk of sICAS progression.

In this study, the low-normal SBP group $(<120 \mathrm{~mm} \mathrm{Hg})$ showed a non-significant pattern towards higher odds of sICAS progression, when compared to the reference group (120 to $139 \mathrm{~mm} \mathrm{Hg}$ ), the finding of which was similar to that by $10-$ $\mathrm{mm} \mathrm{Hg}$ strata of mean SBP, shown in Supplementary Table 4. It is not clear why and how low SBP of $<120 \mathrm{~mm} \mathrm{Hg}$ might be associated with sICAS progression. The J-shaped pattern of association between SBP and sICAS progression, shown in Figure 2 , accords with a previous study ${ }^{13}$ that the low-normal SBP range $(<120 \mathrm{~mm} \mathrm{Hg})$ during follow-up was associated with an increased risk of recurrent stroke. However, since the small sample size was less than 30 and the $\mathrm{Cl}$ was wide in the low-normal SBP group, our finding cannot be yet conclusive until verification with a large sample-sized study.

Our study showed no significant difference in the sICAS progression between normal to high-normal SBP group and high SBP group, the finding of which conflicts with the finding that higher BP of $>140 / 90 \mathrm{~mm} \mathrm{Hg}$ was associated with an increased the risk of stroke in the sICAS territory and stroke overall. This null association between high SBP group and slCAS progression might be due to the beneficial effect from significant BP reduction, which is shown in Supplementary Table 3 .

Contrary to sICAS progression, very-high SBP group was not significantly linked to an increased risk of ischemic stroke in unadjusted analysis (HR 4.7, 95\% Cl 0.88-25.34). This lack of power might have been due to relatively fewer stroke events (12 in total) in a small number of patients, particularly in the very-high SBP group.

In a post hoc analysis of WASID cohort, patients achieving systolic BP $<140 \mathrm{~mm} \mathrm{Hg}$ and total cholesterol level $<200 \mathrm{mg} / \mathrm{dL}$ were less likely to have ischemic stroke and a composite of major vascular event during a 2-year follow-up. ${ }^{16}$ Based on the WASID cohort observation, the Stenting and Aggressive Medical Management for Prevention of Recurrent Stroke in Intracranial Stenosis (SAMMPRIS) study implemented aggressive 
control of risk factors, thereby leading to lower vascular event rates than expected in the medical treatment arm. ${ }^{17}$ In a recent prospective observational study involving 50 acute stroke patients with sICAS $\geq 70 \%$, intensive medical therapy for 12 months with therapeutic targets as low-density lipoprotein cholesterol $\leq 70 \mathrm{mg} / \mathrm{dL}$; $\mathrm{HbA} 1 \mathrm{c} \leq 6.5 \%$; and SBP $\leq 140 \mathrm{~mm} \mathrm{Hg}$ resulted in sICAS regression in 49\%; quiescent sICAS in 43\%; and sICAS progression in $8 \%$ of the patients (vs. $27.1 \% ; 60.0 \%$; and $12.9 \%$, respectively in our study). ${ }^{15}$ The potential factors for the varied results between our sub-analysis of the TOSS-2 trial ${ }^{11}$ and that study ${ }^{15}$ include treatment period (7 months vs. 12 months), statin use (70\% vs. 100\%), antihypertensive use (78\% vs. $>90 \%$ ), degree of sICAS (variable vs. $\geq 70 \%$ for all), and different patterns of antiplatelet prescription.

This study has limitations. First, this is a post hoc exploratory analysis of a completed randomized trial and was based on SBP measures that were averaged throughout the follow-up period. Therefore, our findings cannot provide a causal relationship between SBP levels and SICAS progression. Second, there were a small number of patients in the SBP groups, especially the low-normal SBP group (less than 30 ) and the veryhigh SBP group (less than 20), which resulted in wide confidence intervals, affecting the precision of estimates for sICAS progression and ischemic stroke, respectively. Finally, the exclusion of 55 subjects owing to missing data, especially for the follow-up MRA, might have influenced the current results. Despite the aforementioned limitations, our study is strengthened by the results showing a relationship between the higher SBP levels and the risk of sICAS progression, a potent surrogate marker for stroke recurrence.

\section{Conclusions}

This study showed an independent association of higher SBP level with sICAS progression among individuals after an ICAS stroke. It should be noted that our results should not be interpreted that SBP itself is the most crucial predictor of sICAS progression, but a key component in a global management strategy to mitigate sICAS progression. Given the J-shaped pattern of the association between SBP level and stroke events, ${ }^{13}$ our results on sICAS progression need to be strictly validated through a large prospective study design. This study showed no significant difference of sICAS progression between normal to high-normal SBP and high SBP groups. However, as higher SBP level $\geq 140 \mathrm{~mm} \mathrm{Hg}$ was linked to an increased risk of recurrent stroke among patients with noncardioembolic or ICAS stroke, ${ }^{13,16}$ our results support aiming for consistent SBP levels of $<140 \mathrm{~mm} \mathrm{Hg}$ among individuals after a recent ICAS stroke. ${ }^{3}$

\section{Supplementary materials}

Supplementary materials related to this article can be found online at https://doi.org/10.5853/jos.2017.00136.

\section{References}

1. Wong LK. Global burden of intracranial atherosclerosis. Int J Stroke 2006;1:158-159.

2. Gorelick PB, Wong KS, Bae HJ, Pandey DK. Large artery intracranial occlusive disease: a large worldwide burden but a relatively neglected frontier. Stroke 2008;39:2396-2399.

3. Holmstedt CA, Turan TN, Chimowitz MI. Atherosclerotic intracranial arterial stenosis: risk factors, diagnosis, and treatment. Lancet Neurol 2013;12:1106-1114.

4. Arenillas JF, Molina CA, Montaner J, Abilleira S, González-Sánchez MA, Alvarez-Sabin J. Progression and clinical recurrence of symptomatic middle cerebral artery stenosis: a long-term follow-up transcranial doppler ultrasound study. Stroke 2001;32:2898-2904.

5. Wong KS, Li H, Lam WW, Chan YL, Kay R. Progression of middle cerebral artery occlusive disease and its relationship with further vascular events after stroke. Stroke 2002;33:532-536.

6. Kasner SE, Chimowitz MI, Lynn MJ, Howlett-Smith H, Stern BJ, Hertzberg VS, et al. Predictors of ischemic stroke in the territory of a symptomatic intracranial arterial stenosis. Circulation 2006;113:555-563.

7. Turan TN, Cotsonis $G$, Lynn MJ, Chaturvedi S, Chimowitz M; Warfarin-Aspirin Symptomatic Intracranial Disease (WASID) Trial Investigators. Relationship between blood pressure and stroke recurrence in patients with intracranial arterial stenosis. Circulation 2007;115:2969-2975.

8. Rothwell PM, Howard SC, Spence JD; Carotid Endarterectomy Trialists' Collaboration. Relationship between blood pressure and stroke risk in patients with symptomatic carotid occlusive disease. Stroke 2003;34:2583-2590.

9. Sutton-Tyrrell K, Wolfson SK Jr, Kuller LH. Blood pressure treatment slows the progression of carotid stenosis in patients with isolated systolic hypertension. Stroke 1994;25:4450.

10. Reed DM, Resch JA, Hayashi T, MacLean C, Yano K. A prospective study of cerebral artery atherosclerosis. Stroke 1988;19:820-825.

11. Kwon SU, Hong KS, Kang DW, Park JM, Lee JH, Cho YJ, et al. Efficacy and safety of combination antiplatelet therapies in patients with symptomatic intracranial atherosclerotic stenosis. Stroke 2011:42:2883-2890.

12. Chobanian AV, Bakris GL, Black HR, Cushman WC, Green LA, 
Izzo JL Jr, et al. The seventh report of the joint national committee on prevention, detection, evaluation, and treatment of high blood pressure: the JNC 7 report. JAMA 2003;289:25602572.

13. Ovbiagele B, Diener HC, Yusuf $S$, Martin RH, Cotton D, Vinisko $R$, et al. Level of systolic blood pressure within the normal range and risk of recurrent stroke. JAMA 2011;306:21372144.

14. Lemeshow S, Hosmer DW Jr. A review of goodness of fit statistics for use in the development of logistic regression models. Am J Epidemiol 1982;115:92-106.
15. Leung TW, Wang L, Soo YO, Ip VH, Chan AY, Au LW, et al. Evolution of intracranial atherosclerotic disease under modern medical therapy. Ann Neurol 2015;77:478-486.

16. Chaturvedi S, Turan TN, Lynn MJ, Kasner SE, Romano J, Cotsonis $G$, et al. Risk factor status and vascular events in patients with symptomatic intracranial stenosis. Neurology 2007;69:2063-2068.

17. Chimowitz MI, Lynn MJ, Derdeyn CP, Turan TN, Fiorella D, Lane BF, et al. Stenting versus aggressive medical therapy for intracranial arterial stenosis. N Engl J Med 2011;365:9931003. 
Supplementary Table 1. Severity of baseline sICAS across SBP category

\begin{tabular}{|c|c|c|c|c|c|}
\hline \multirow{2}{*}{ sICAS severity } & \multicolumn{4}{|c|}{ Mean SBP category (mm Hg) } & \multirow{2}{*}{$P$} \\
\hline & $<120(n=28)$ & 120 to $139(n=233)$ & 140 to $159(n=123)$ & $\geq 160(n=18)$ & \\
\hline Mild $(<50 \%)$ & $6(21.4)$ & $81(34.8)$ & $40(32.5)$ & $7(38.9)$ & 0.509 \\
\hline Moderate (50\% to $69 \%$ ) & $9(32.1)$ & 75 (32.2) & $43(35.0)$ & $9(50.0)$ & \\
\hline Severe (70\% to $99 \%)$ & $13(46.4)$ & 74 (31.8) & $38(30.9)$ & $2(11.1)$ & \\
\hline Occlusion & $0(0)$ & $3(1.3)$ & $2(1.6)$ & $0(0)$ & \\
\hline
\end{tabular}

Values are presented as numbers (\%) of patients.

sICAS, symptomatic intracranial atherosclerotic stenosis; SBP, systolic blood pressure. 
Supplementary Table 2. Distribution of baseline sICAS across SBP category

\begin{tabular}{|c|c|c|c|c|c|}
\hline \multirow{2}{*}{ Site of sICAS } & \multicolumn{4}{|c|}{ Mean SBP category $(\mathrm{mm} \mathrm{Hg})$} & \multirow{2}{*}{$P$} \\
\hline & $<120(n=28)$ & 120 to $139(n=233)$ & 140 to $159(n=123)$ & $\geq 160(n=18)$ & \\
\hline Right MCA & $10(35.7)$ & $102(43.8)$ & $41(33.3)$ & $5(27.8)$ & 0.128 \\
\hline Left MCA & $14(50.0)$ & 93 (39.9) & $54(43.9)$ & $6(33.3)$ & \\
\hline BA & $4(14.3)$ & 38 (16.3) & $28(22.8)$ & $7(38.9)$ & \\
\hline
\end{tabular}

Values are presented as numbers (\%) of patients.

sICAS, symptomatic intracranial atherosclerotic stenosis; SBP, systolic blood pressure; MCA, middle cerebral artery; BA, basilar artery. 
Supplementary Table 3. Comparisons of SBP between baseline and follow-up by each mean SBP category

\begin{tabular}{lcccc}
\hline & \multicolumn{4}{c}{ Mean SBP category $(\mathrm{mm} \mathrm{Hg})$} \\
\cline { 2 - 6 } & Low-normal $(<120)$ & Normal to high-normal (120 to 139) & High (140 to 159) & Very-high $(\geq 160)$ \\
\hline SBP $(\mathrm{mm} \mathrm{Hg})$ & $121.5 \pm 14.0$ & $133.1 \pm 15.8$ & $154.7 \pm 22.8$ & $175.4 \pm 27.0$ \\
At baseline & $112.8 \pm 11.0$ & $126.6 \pm 12.6$ & $143.9 \pm 15.6$ & $167.8 \pm 26.1$ \\
At follow-up & 0.470 & 0.215 & 0.002 & 0.806 \\
$P^{*}$ & & & & \\
\hline
\end{tabular}

Values are presented as mean \pm standard deviation.

SBP, systolic blood pressure.

*B By paired $t$-test. 
Supplementary Table 4. ORs for the progression of sICAS and recurrent ischemic stroke by 10-mm Hg strata of mean SBP

\begin{tabular}{|c|c|c|c|c|c|c|}
\hline & \multicolumn{6}{|c|}{ 10-mm Hg strata of mean SBP $(\mathrm{mm} \mathrm{Hg})$} \\
\hline & $\begin{array}{l}<120^{*} \\
(n=28)\end{array}$ & $\begin{array}{c}120 \text { to } 129 \\
(n=102)\end{array}$ & $\begin{array}{c}130 \text { to } 139 \\
(n=131)\end{array}$ & $\begin{array}{c}140 \text { to } 149 \\
(n=84)\end{array}$ & $\begin{array}{c}150 \text { to } 159 \\
(n=39)\end{array}$ & $\begin{array}{l}\geq 160^{+} \\
(n=18)\end{array}$ \\
\hline Primary outcome & \multicolumn{6}{|c|}{ OR $(95 \% \mathrm{Cl})$} \\
\hline sICAS progression (n [\%]) & $6(21.4)$ & $12(11.8)$ & $13(9.9)$ & $11(13.1)$ & $3(7.7)$ & $7(13.5)$ \\
\hline Unadjusted & $2.48(0.85-7.21)$ & $1.21(0.53-2.78)$ & 1 [Reference] & $1.37(0.58-3.21)$ & $0.76(0.20-2.80)$ & $5.78(1.91-17.48)^{*}$ \\
\hline \multicolumn{7}{|l|}{ Adjusted } \\
\hline Model $I^{\S}$ & $1.91(0.60-6.09)$ & $1.05(0.44-2.52)$ & 1 [Reference] & $1.24(0.49-3.15)$ & $0.63(0.13-3.02)$ & $8.53(2.38-30.62)^{\neq}$ \\
\hline Model II" & $2.04(0.62-6.71)$ & $1.19(0.48-2.94)$ & 1 [Reference] & $1.29(0.48-3.45)$ & $0.79(0.16-3.89)$ & $9.47(2.58-34.73)^{+}$ \\
\hline Secondary outcome & \multicolumn{6}{|c|}{$\mathrm{HR}(95 \% \mathrm{Cl})$} \\
\hline Ischemic stroke (n [\%]) & $0(0)$ & $3(2.9)$ & $3(2.3)$ & $4(4.8)$ & $0(0)$ & $2(11.1)$ \\
\hline Unadjusted & $0.00(0.00-0.00)$ & $1.11(0.22-5.52)$ & 1 [Reference] & $2.31(0.50-10.72)$ & $0.00(0.00-0.00)$ & $7.33(1.15-46.53)^{* *}$ \\
\hline \multicolumn{7}{|l|}{ Adjusted } \\
\hline Model $I^{\S}$ & $0.00(0.00-0.00)$ & $5.86(0.56-61.16)$ & 1 [Reference] & $3.56(0.31-41.24)$ & $0.00(0.00-0.00)$ & $1.63(0.03-95.04)$ \\
\hline Model II" & $0.00(0.00-0.00)$ & $6.96(0.60-80.51)$ & 1 [Reference] & $2.89(0.21-39.06)$ & $0.00(0.00-0.00)$ & $1.40(0.02-84.56)$ \\
\hline
\end{tabular}

sICAS, symptomatic intracranial atherosclerotic stenosis; $\mathrm{SBP}$, systolic blood pressure; $\mathrm{OR}$, odds ratio; $\mathrm{HR}$, hazard ratio; $\mathrm{Cl}$, confidence interval.

*Includes SBP <110 mm Hg (n=3), SBP 110-119 mm Hg ( $n=25) ;{ }^{\dagger}$ Includes SBP 160-169 mm Hg (n=10), SBP 170-179 mm Hg (n=4), SBP 180-189 mm Hg $(n=1)$, SBP 190-199 mm Hg (n=2), and SBP 200-209 mm Hg $(n=1) ;{ }^{*} P<0.005 ;{ }^{5}$ Adjusted for age, male sex, serum levels of creatinine and homocysteine, hypertension, treatment for high BP, renin-angiotensin system modifier use, smoking as model I; "Adjusted for model I plus diabetes mellitus, glycosylated hemoglobin, low-density lipoprotein cholesterol, qualifying stroke severity, cilostazol+aspirin medication (vs. clopidogrel+aspirin), and statin medication; 'Defined as any ischemic stroke or stroke in the sICAS territory; ${ }^{* *} P=0.035$. 
Supplementary Table 5. Multivariable adjusted odds ratios (ORs) of covariates included in the backward elimination regression analyses

\begin{tabular}{lcc}
\hline Covariates & sICAS progression & $P$ \\
\cline { 2 - 2 } & OR (95\% Cl) & \\
\hline Age (1-yr difference) & $0.96(0.93-0.98)$ & 0.003 \\
Qualifying stroke severity & $0.87(0.75-1.01)$ & 0.071 \\
\hline
\end{tabular}

sICAS, symptomatic intracranial atherosclerotic stenosis; $\mathrm{Cl}$, confidence interval. 